\title{
Une mélancolie romantique.Concept de création et création de concept dans Les Chimères de Nerval.
}

\section{Martin Mees}

\section{OpenEdition}

\section{Journals}

Édition électronique

URL : http://journals.openedition.org/lcc/1204

DOI : $10.4000 /$ lcc. 1204

ISSN : 2430-4247

\section{Éditeur}

Université Aix-Marseille (AMU)

\section{Référence électronique}

Martin Mees, « Une mélancolie romantique.Concept de création et création de concept dans Les

Chimères de Nerval. », Les chantiers de la création [En ligne], 9 | 2016, mis en ligne le 28 décembre 2016, consulté le 08 avril 2020. URL : http://journals.openedition.org/lcc/1204 ; DOI : https://doi.org/ $10.4000 /$ lcc. 1204

Ce document a été généré automatiquement le 8 avril 2020.

Tous droits réservés 


\title{
Une mélancolie romantique.Concept de création et création de concept dans Les Chimères de Nerval.
}

\author{
Martin Mees
}

1 À suivre le titre de ce numéro, l'œuvre serait «hantée par le concept ». Ces quelques mots semblent ouvrir à une relecture complète des rapports entre art et philosophie, qui relèveraient peut-être moins d'une concurrence entre pratiques, que d'une hantise de la création artistique par les idées de la philosophie venant habiter l'œuvre, consciemment ou non. Une relecture qui nous conduit immédiatement à de nouvelles perspectives de recherche: ces concepts "fantômes» dévoilent-ils une certaine « vérité » de l'œuvre ou, à tout le moins, des substrats philosophiques régissant sa création? Car s'il y a du concept au fondement de l'œuvre, la recherche et l'identification du ou des concept(s) présidant à la constitution de telle ou telle œuvre permettrait peut-être d'en dégager, au-delà des motifs épars, une raison d'être, une unité philosophique éclairant à la fois l'urgence à la source de la création, c'est-à-dire la question qui travaillait l'artiste, et à la fois la forme, toujours singulière, de l'œuvre : en somme, esquisser une piste de réponse à la question "pourquoi cette forme-là ?». En outre, un tel postulat impliquerait sans doute de considérer l'ensemble « œuvreconcept » comme un tout qui interagirait en deux sens : si le concept nous permet une autre compréhension de l'œuvre, à son tour l'œuvre travaille simultanément le concept en l'incarnant dans une forme singulière. En lui donnant corps dans un autre contexte que celui de la philosophie, elle ne peut manquer de développer l'idée, la préciser, la questionner. De là, nous pouvons tirer deux principes méthodologiques qui seront au départ de cette analyse et constitueront aussi son enjeu. D'une part, il semble envisageable de comprendre les productions de l'art par une approche philosophique, à même peut-être d'éclairer la dimension conceptuelle travaillant l'œuvre en sous-main. D'autre part, l'œuvre ne serait pas uniquement une présentation, une illustration de concepts mais, bien plus, un lieu propice au travail des idées, des concepts, qui viendraient s'y réfléchir. 
2 À travers le terme d' «œuvre» est évoquée la spécificité de l'art, sa dimension singulière, originale, tenant dans une forme précise qui agence le sens d'une manière qui n'est ni modifiable, ni répétable, ni transposable. Or, si c'est bien cela qu'il s'agit d'étudier ici, il me semble difficile de le faire sans partir d'une telle singularité, condition nécessaire et préalable à tout discours théorisant. Non que nous ne pourrions ici nous abstraire de l'œuvre, mais sans doute faut-il penser au départ de celle-ci, afin que le discours et son objet entrent en résonance, en cohérence : c'est-à-dire penser avec l'objet, lui-même pensant, et sans surplomb. C'est donc à travers un concept singulier, situé dans une œuvre précise, que nous évaluerons nos hypothèses : celui de mélancolie dans le recueil Les Chimères de l'écrivain romantique Gérard de Nerval. Ce choix n'est pas arbitraire, il relève d'une nécessité triple prenant racine dans la question que nous voulons traiter.

3 Nerval, en premier lieu, - et sans nécessairement l'expliciter d'ailleurs - est habité par la question des rapports entre littérature et philosophie qu'il hérite du romantisme d'Iéna. De manière plus générale, cet auteur se trouve au croisement de différentes traditions littéraires et philosophiques, françaises comme allemandes, qu'il réinvestit dans son œuvre de façon très riche, avec la volonté bien romantique (héritée sans doute de Schiller) de faire de l'art le medium d'une transformation de soi et du monde en y déployant une véritable pensée. La mélancolie, quant à elle, est un concept foisonnant, dont les premières traces en philosophie remontent à Aristote, traversent toute la tradition médiévale, baroque et se poursuivent encore bien après le romantisme. Loin d'être une notion univoque, la mélancolie peut désigner tout à la fois une vague tristesse, une humeur mortifère, un excès d'imagination, une prédisposition au génie, une puissance de folie ou encore un certain plaisir esthétique. Il semble intéressant d'examiner à nouveaux frais un concept aussi riche et aussi polymorphe, d'autant plus chez un romantique comme Nerval. Car si tout le monde s'accorde pour affirmer que le romantisme est traversé de mélancolie, elle demeure souvent impensée, considérée simplement comme le ton nostalgique ou le vague à l'âme diffus d'un courant jugé parfois plaintif à l'excès. Il y a donc un double intérêt à s'attarder sur cette notion, tant pour le concept en tant que tel, que pour ce qu'il nous apprend d'une certaine poétique, intérêt qui s'augmente encore lorsqu'on pense à l'affinité profonde de la mélancolie avec l'idée d'une hantise ou d'une hantologie. La mélancolie, en effet, c'est « le passé qui ne passe pas» (Kristeva 70) et qui revient dans l'imagination, ce qui suggère, nous le verrons, un mode de pensée ou de création qui se comprendrait sur le mode de la revenance. Enfin, l'œuvre Les Chimères (recueil de huit poèmes de Nerval, publié à la fin de sa vie, en 1854) semble s'imposer pour une telle enquête, par ses références explicites à la mélancolie et par la profondeur métaphysique des sonnets qui la composent, profondeur annoncée par leur auteur et confirmée par la masse extraordinaire de littérature critique existant sur ces compositions assez obscures.

4 Avant d'entamer l'analyse en tant que telle, il nous faut encore préciser qu'il ne s'agira pas de proposer ici une interprétation complète des Chimères, ni même d'un seul de ses poèmes, ce qui exigerait des développements considérables et nous éloignerait de l'enjeu propre à cette étude. Nous construirons cette dernière en deux parties: la première tentera de dégager les traits principaux de la mélancolie nervalienne, c'est-àdire ce que Les Chimères font de ce concept. La seconde visera à montrer comment la mélancolie, comme concept, éclaire l'écriture de Nerval et jusqu'à sa conception implicite de la création poétique. 


\section{Le concept de mélancolie}

5 Attachons-nous dans un premier temps à saisir la présentation de la mélancolie dans les compositions nervaliennes. Les Chimères s'ouvrent sur l'un des poèmes les plus connus de Nerval, «El Desdichado », qui semble avoir une dimension programmatique par rapport au reste du recueil mais également par rapport au traitement nervalien de la mélancolie qui s'y décline en deux dimensions complémentaires (ainsi que l'a bien vu Pierre Laforgue $\left.{ }^{1}\right)$ : un motif particulièrement fort, celui du Soleil noir, et une structure poétique, l'une des lignes de force de l'œuvre, celle d'une perte, originaire et fondamentale.

6 Je suis le Ténébreux, - le Veuf, - l'Inconsolé, Le Prince d'Aquitaine à la Tour abolie :

Ma seule Etoile est morte, - et mon luth constellé

Porte le Soleil noir de la Mélancolie. (III, 645)

7 Cette perte connaît plusieurs régimes dès le premier vers : le deuil (le «je » est veuf d'une étoile annoncée comme morte), le déshéritage (le titre "El desdichado » étant une reprise de l'Ivanhoé de Walter Scott qui comprend ce terme comme "le déshérité »), la privation (le "ténébreux » est sans lumière), sur un mode définitif et irrémédiable (il est aussi «inconsolé »). Quel est exactement l'objet de cette perte, quelle est cette étoile au tombeau? La suite des Chimères, et plus généralement l'ensemble de l'œuvre nervalienne, développe cette perte qui prend de multiples visages.

8 Il y a en premier lieu, et d'un point de vue biographique, la perte pour Nerval de la mère qu'il n'a jamais connue et qui hante l'entièreté de ses productions, constituant, comme l'a bien montré Dagmar Wieser, le moteur initial d'une écriture qui apparaît parfois comme la tentative de surmonter ce manque originaire. Celui-ci se traduit dans la logique nervalienne par une perte plus fondamentale et métaphysique, celle d'une transcendance, d'un garant du sens, de Dieu, qui chez Nerval prend le plus souvent la figure d'Isis, divinité orientale et maternelle ${ }^{2}$. Reprenant de l'Allemand Jean-Paul l'idée de la mort de Dieu, Nerval développe cette perte dans l'avant-dernier poème des Chimères, « Le Christ aux oliviers » où apparaît aussi le motif du soleil noir :

Le dieu manque à l'autel où je suis la victime

10 Dieu n'est pas, Dieu n'est plus! Mais ils dormaient toujours!

$11 \quad[. .$.

12

Partout le sol désert côtoyé par des ondes,

Des tourbillons confus d'océans agités...

Un souffle vague émeut les sphères vagabondes,

Mais nul esprit n'existe en ces immensités.

En cherchant l'œil de Dieu, je n'ai vu qu'un orbite Vaste, noire et sans fond, d'où la nuit qui l'habite Rayonne sur le monde et s'épaissit toujours ; [...] (III, 649)

13 Si pour Freud la mélancolie est le symptôme net d'un deuil impossible de la figure maternelle, elle prend chez Nerval une dimension métaphysique, corroborée également 
dans Aurélia. Le narrateur, qui y mène la quête impossible d'Isis, est confronté à un soleil noir, et cela juste après avoir posé que pour lui, « Dieu c'est le soleil » (III, 731). L'assimilation « Dieu-mère » se voit encore additionnée un troisième terme, amoureux, déjà présent en Isis qui est aussi l'épouse, comme le poème « Horus » le rappelle (III, 646). Dans l'histoire de l'auteur, il s'agit d'une actrice, Jenny Colon, dont il fut véritablement épris et qui mourut prématurément; en littérature, il s'agit d'un amour complètement idéalisé pour une figure féminine portant mille masques (Aurélia, Sylvie, Adrienne...) et demeurant toujours inaccessible au héros des romans. À l'instar de Novalis qui cherchait Sophie dans la fleur bleue cachée derrière les voiles d'Isis, Nerval poursuit la même quête d'une rose, d'un myosotis ou d'une étoile finalement indévoilable, inatteignable, fauchée par la mort avant même d'avoir pu être touchée, et le plongeant dans ce deuil généralisé, exprimé encore dans « Le Christ aux oliviers »: c'est la perte plus généralisée du sens qui advient, le désenchantement d'une vie qui sombre dans la mélancolie. Le soleil est éclipsé et la lueur de l'étoile, plus faible déjà, a elle-même disparu.

17 Cette perte de l'Autre affecte ultimement le Moi, qui dans cette absence de signifiance ne parvient plus à construire une identité qui fasse sens. En perdant l'Autre, il se perd lui-même, et assez paradoxalement, il se perd en l'autre. «El Desdichado » n'est qu'une longue énumération d'un « je » qui ne parvient plus à saisir sa propre unité, qui tente de convoquer des figures extérieures auxquelles se rattacher, un sens extérieur à se réapproprier, mais cette tentative d'identification, multipliée sans fin, ne fait que parachever sa dislocation. Le " je » se perd dans une énumération non hiérarchisée, sans ordre, si bien qu'ultimement la mélancolie apparaît comme cet espace d'asymbolie généralisée ou le Moi perd l'altérité et se dissout simultanément en elle (ce qui rappelle aussi la prégnance du thème du double dans l'écriture nervalienne, incarnation à la fois du même et de l'autre, qui pourchasse le narrateur). On peut voir chez Nerval les échos de ce que Christine Buci-Glucksmann définit comme l'idée moderne de mélancolie, à la fois deuil impossible et ratage : le sens est toujours déjà perdu, et l'écrit vient toujours trop tard. Dans ce «trop tard", la subjectivité se dissout, elle est en fuite, plurielle, disjointe, en quête perpétuelle d'un monde qui n'existe plus. À l'instar de Don Quichotte, « le sujet s'abîme dans le "culte des images", dans toutes les visions et folies propres à la mélancolie depuis l'Antiquité » (Buci-Glucksmann 38).

19 Cependant, ce qui se dessine dans ce mode mélancolique de l'écriture nervalienne, ce n'est pas à proprement parler une absence pure, un néant de sens. Le soleil noir n'est pas simplement un trou béant, c'est une négativité. Il ne s'agit certes plus d'une présence débordante, pleine, positive, plutôt de son éclipse, de son contour, qui présente toujours le soleil, mais par la négative, au sens d'un négatif photographique, qui dit le plein par le vide, l'excès par le creux, la présence par l'absence. La mélancolie se pose donc comme un régime de sens inverse, critique, paradoxal et oxymorique, mais n'est pas un nihilisme. Cette idée rejoint les développements de Rousseau sur une mélancolie qu'on a pu qualifier d'«expansive», étant cet "espace du désir qui se creuse, qui se tend au-delà de ce que la réalité (dans la sensation) ou l'imagination (dans la rêverie) peut donner " (Raybaud 46). La mélancolie grandit alors tant dans le 
manque que dans un excès tout aussi inassouvissable, ainsi que le professe le René de Chateaubriand ${ }^{3}$. Chez Nerval, la mélancolie ne s'enfoncera pas dans un désespoir sans fond mais connaitra une reprise, opérée par l'art. Le soleil noir est porté par un luth qui a la particularité d'être constellé, c'est-à-dire habité, accompagné de la dimension stellaire qui semble faire défaut au «je » du poème. Qu'est-ce que cela signifie? Le soleil noir a bel et bien des rayons, il produit une lumière, bien que ce soit sur un mode négatif, un mode d'existence qui est peut-être celui de la poésie elle-même en ce qu'elle se distingue de toute appréhension positive du réel - et l'on sait l'insistance avec laquelle Nerval tente de relativiser les assises d'une rationalité scientiste et matérialiste par son écriture du voyage, de l'occulte ou encore de la folie (Brix 180-2). Investie d'une capacité à recréer du sens par son rayonnement singulier, la poésie nervalienne semble riposter au désenchantement par un texte pensé comme chant, à même de chanter et réen-chanter comme l'annonce le poème " Delfica »:

l'investir entièrement en art pour revaloriser et ramener à la présence un sens absent. Comme le note Julia Kristeva : «L'introspection de Nerval semble indiquer que nommer la mélancolie le situe au seuil d'une expérience cruciale : à la crête entre apparition et disparition, abolition et chant, non-sens et signes " (163). Ce réenchantement semble d'ailleurs contenir la conclusion des Chimères, dans le sonnet « Vers Dorés », où les vers (qui brillent de la couleur du soleil) annoncent un réinvestissement du monde par le souffle du divin, ce qui passe notamment par le langage, « le verbe » :

Crains dans le mur aveugle un regard qui t'épie

A la matière même un verbe est attaché ...

Ne la fais pas servir à quelque usage impie !

Souvent dans l'être obscur habite un Dieu caché ;

Et comme un œil naissant couvert par ses paupières, Un pur esprit s'accroît sous l'écorce des pierres! (III, 651)

27 À l'orbite vide du « Christ aux oliviers », les « Vers dorés » opposent un œil naissant qui affirme, dans la nuit d'un monde matériel déserté, la présence d'un principe divin habitant « l'être obscur ». C'est bien par l'intermède d'un verbe poétique que semble se révéler ce «pur esprit» qui hante le réel. Kristeva distingue deux caractéristiques majeures de la mélancolie : la perte de la Chose, de l'idéal, ce qui se traduit par une disparition du lien, et le désinvestissement du langage, du signifiant, auquel on ne croit plus. Il semble que le geste nervalien soit justement une plongée à la poursuite du signifiant, qui s'échappe toujours, pour ramener, non pas la Chose, mais son reflet, son rêve, son image, comme nous le suggère « Horus » :

La déesse avait fui sur sa conque dorée

La mer nous renvoyait son image adorée 
Par une autre voie, Nerval rejoint peut-être l'idée médiévale selon laquelle la mélancolie représente un débordement de l'imagination, une absorption complète dans l'abîme de l'image, au détriment de toute spéculation in abstracto ${ }^{4}$. Mais si la mélancolie entrave la pensée du métaphysicien, elle semble revalorisée par le poète, qui y trouve à la fois le moteur de sa création et l'enjeu de son écriture. En effet, la mélancolie se présente dans Les Chimères comme ce régime singulier d'une imagination prise dans un mouvement dialectique entre perte et restauration. Une mélancolie qui, dans cette tension de l'imagination, invente peut-être une autre modalité de « l'apparaître », celle d'une représentation moderne puisant dans les ressources de la négativité d'un soleil noir pour tenter de dire, de montrer, de faire advenir du sens. Pour reprendre la formule de Madame de Staël, " l'imaginaire mélancolique rend heureux un moment, en faisant rêver l'infini » (360) : c'est dans la mélancolie que l'infini, négatif du fini, réserve de possible, peut se rêver, moyennant le chant du poète. C'est en s'imaginant que la poésie nervalienne pense, qu'elle se réfléchit dans sa forme précisément comme image, et qu'elle réfléchit par-là ses propres termes, ses propres concepts. La mélancolie, qu'on a aussi pu qualifier d' «œil de la pensée ${ }^{5}$, en fait partie et occupe même une place cardinale dans l'entreprise poétique de Nerval, par cet espace (caractéristiquement romantique) qu'elle ouvre pour l'existence du poète mais surtout pour son art. Car il s'agit bien, avec la mélancolie, d'une autre conception de l'art, de sa destination comme des conditions de sa création. C'est ce dernier point que nous voulons maintenant préciser, en analysant comment la mélancolie peut éclairer notre compréhension de la démarche poétique de Nerval.

\section{Une création chimérique}

La création littéraire, telle que la pense et la pratique Nerval, que ce soit dans ses imitations de poètes étrangers ou anciens, mais aussi dans ses nouvelles, peut se lire comme un mouvement de reprise d'un héritage poétique auquel l'auteur fait accomplir un écart ; c'est dans cette dernière opération que semble résider pour le romantique l'originalité propre au créateur. Dans son introduction à Faust, Nerval traduit l'activité du poète en ces termes qui font écho à la « descente aux enfers » que Gérard accomplit dans Aurélia, et à son association à Orphée dans « El Desdichado " : " Il faut aller poser le pied solidement sur le monde ancien, pénétrer dans le monde des fantômes, prendre part à sa vie pour quelque temps, et trouver les moyens de lui ravir l'ombre d'Hélène, pour la faire vivre matériellement dans notre atmosphère. Ce sera là presque la descente d'Orphée » $(\mathrm{I}, 508)$. Les textes de Nerval, par leurs références explicites à d'autres auteurs, se présentent eux-mêmes comme des accouplements inattendus d'œuvres antérieures. Aurélia, par exemple, mêle les croyances et les époques en s'inspirant simultanément d'Apulée, de Dante et de l'ésotérique Swedenborg, pour constituer un « roman-vision à la Jean-Paul » que Nerval qualifiera encore de «millepattes romantique ", où la combinaison n'est plus simplement double mais tend à la multiplicité. Créer pour Nerval c'est toujours recréer, reprendre dans le passé : « inventer au fond c'est se ressouvenir » (III, 451).

33 Le terme de " chimère » est lui-même un mot au sens double, reflétant au sens figuré le sens propre de la nature composite de l'animal. À la fois créature mythologique et illusion, elle présente à elle seule, comme le souligne Bertrand Marchal, « le processus 
historique qui oppose une antiquité perçue comme le temps des mythes vrais et efficaces à une modernité qui identifie le mythe à l'irréel » (418). Nerval note dans les Illuminés que « toute religion qui tombe dans le domaine des poètes se dénature bientôt, et perd son pouvoir sur les âmes » (II, 1079), aussi la chimère maintient-elle vive la trace de cette chute. Elle atteste par-là du désir poétique de restauration d'une forme de pouvoir quasi-religieux de l'art, à mettre en parallèle avec l'amour-religion dont il rend compte, comme nous le rappelle encore Marchal : « La chimère, dragon vaincu ou volcan éteint, porte ainsi le rêve d'un réveil du feu, rêve qui conjoint le retour du paganisme et la révolte contre l'ordre établi, la subversion du monde d'en haut par le monde d'en bas, par la vertu d'un feu qui n'est rien d'autre que le correspondant cosmique du feu amoureux » (409).

34 L'association, proposée dans toute l'œuvre de Nerval, du christianisme et du paganisme semble d'ailleurs concrétiser ce message du poète, une restauration de la spiritualité qui se pense moins comme retour que comme création de formes neuves dans un avenir non encore advenu. Mais ces formes neuves n'apparaissent qu'au prix du mélange d'éléments préexistants, comme la chimère elle-même se compose d'animaux réels : pas d'invention ex nihilo, seule leur fusion est originale. Il en va de même pour le poète, qui ne crée que par composition et re-composition. Cela se remarque à divers niveaux dans Les Chimères, depuis les références explicites à des penseurs dont Nerval s'inspire (Hegel, Jean-Paul, Pythagore), jusqu'à la réponse métaphysique qu'il avance à la fin de «El Desdichado », annonçant les « Vers dorés » :

Et j'ai deux fois vainqueur traversé l'Achéron :

Modulant tour à tour sur la lyre d'Orphée

Les soupirs de la Sainte et les cris de la Fée. (III, 645)

Si l'on veut traverser vainqueur et vivant l'Achéron, il s'agit de moduler « tour à tour sur la lyre d'Orphée » les chrétiens soupirs de la sainte et les cris païens des fées. Les chimères habitent tous les niveaux de l'écriture, qu'elles soient symboliques, philosophiques, sonores ou même végétales. À titre d'exemple, un peu plus haut dans «El Desdichado»:

Dans la nuit du Tombeau, Toi qui m'as consolé,

Rends-moi le Pausilippe et la mer d'Italie,

La fleur qui plaisait tant à mon cœur désolé,

Et la treille où le Pampre à la Rose s'allie. (III, 645)

Cette «fleur qui plaisait tant à mon cœur désolé » et « la treille où le pampre à la rose s'allie " sont des signes chimériques de recouvrement du sens : l'étoile ressuscite en fleur et la treille est la métaphore d'un réseau où pampre bacchique et rose mystique s'unissent. Cette fleur se retrouve au poème suivant dans le personnage de Myrtho, mélange végétal, lorsque Nerval conclut : «Le pâle Hortensia s'unit au Myrthe vert » mais aussi sonore, Myrtho apparaissant comme la fusion de Myrthe et d'Hortensia. Le myosotis apparaît aussi de manière récurrente, associé à un " doux langage » (III, 746), celui de la poésie, mais aussi à ce refrain, «ne m'oubliez pas!» (III, 746), qui est encore une façon de vouloir tenir le lien, l'amour, à travers la mémoire dont le myosotis est le symbole : il s'agit, pour le poète, de commémorer ${ }^{6}$ le deuil de l'étoile ou de la fleur, en multipliant les images combinatoires florales. Il n'est pas anodin que le narcisse trouve également sa place au jardin mélancolique de Nerval, rappelant celui qui trouva la consolation en se noyant dans sa propre image... Les exemples de ce type abondent dans Les Chimères, consacrant une poétique qui ne peut déployer son sens qu'à travers 
une création sur le mode de l'association, de l'analogie, du réseau, et ce, à tous les niveaux de sa forme. La mélancolie et ce qu'elle nous dévoile de l'écriture nervalienne sont donc consubstantiels à la forme même de la poétique des Chimères. Pour le dire avec Julia Kristeva :

Par un saut dans l'univers orphique de l'artifice (de la sublimation), le ténébreux ne retient de l'expérience et de l'objet traumatiques du deuil qu'une sonorité lugubre ou passionnelle. Il touche ainsi, par les composantes mêmes du langage, à la Chose perdue. Son discours s'identifie à elle, l'absorbe, la modifie, la transforme : il sort Eurydice de l'enfer mélancolique et lui redonne une existence nouvelle dans son chant-texte. [...] en fin de compte, et par-delà leur valeur idéologique, le poème intègre ces anaphores au titre de signes sans signifié, d'infra-, de supra-signes qui, par-delà la communication, essaient de toucher l'objet mort ou intouchable, de s'approprier l'être innommable. (171-5)

Mais cette quête mystique n'est pas à prendre au sens propre : il s'agit d'une fiction poétique, d'une chimère, qui ne possède aucun pouvoir «supra-naturel» - pour reprendre le vocabulaire de Nerval - mais n'en est pas moins signifiante pour autant. Ce n'est même que comme illusion, seconde dimension de la chimère, que la poésie nervalienne trouve les conditions de sa performativité. L'art est une illusion lucide, une image qui assume sa dimension d'irréalité, et Nerval affirme à diverses reprises «préférer» ou "regretter» les illusions perdues avec la modernité. La poésie nervalienne donne suite à un désenchantement qui nous « déshérite » (II, 237) de nos illusions premières et choisit, en connaissance de cause, de reproduire une illusion qui ne semble pas moins signifiante que toute la science du siècle. Aussi, dans le poème "Ode » (ou "Le temps") datant du début de la carrière poétique de Nerval, sont déjà exprimés clairement l'association de l'idéal poétique à la chimère, à l'illusion, voire à l'erreur, et ce n'est sans doute pas un hasard si cette conception de l'art s'épanouit précisément dans un poème questionnant le rapport à la temporalité :

« Le bonheur n'est point sur la terre,

Votre amour n'est qu'une chimère,

Si l'art est ultimement chimère, c'est parce que tout absolu positif demeure hors de portée de l'homme et finalement ne se révèle pas signifiant pour le poète : «L'humble vérité n'a pas les ressources immenses des combinaisons dramatiques ou romanesques. [...] la réalité grimace à côté du mensonge » (II, 271). Le geste des Chimères sera d'affirmer ce caractère de l'art: Nerval ne vit pas l'imaginaire comme une illusion tempérée mais comme une illusion totale qui, en retour, déréalise le monde pour n'y laisser que l'acte créatif du poète, «la dernière folie » de Nerval étant « de se croire poète » (III, 458). 
52 La logique chimérique, combinatoire, est donc virtuellement infinie, modèle poétique d'une modernité elle-même hybride et en quête de son propre sens, comme nous la décrit Nerval dans « Sylvie » :

Nous vivions alors dans une époque étrange, comme celles qui d'ordinaire succèdent aux révolutions ou aux abaissements des grands règnes. [...] c'était un mélange d'activité, d'hésitation et de paresse, d'utopies brillantes, d'aspirations philosophiques ou religieuses, d'enthousiasmes vagues, mêlés de certains instincts de renaissance; d'ennuis des discordes passées, d'espoirs incertains, - quelque chose comme l'époque de Pérégrinus et d'Apulée. L'homme matériel aspirait au bouquet de roses qui devait le régénérer par les mains de la belle Isis. (III, 538)

Cette attente de l'époque semble trouver sa relève dans le projet d'une certaine poésie romantique, non celle des poètes mages ou prophètes, mais celles des poètes qui n'arriveront jamais à l'être. Si Nerval se figure bien comme le gardien du feu sacré et revendique le «titre de vestal [sic] » (III, 900), il n'y a pas chez lui l'orgueil du poète se représentant sur sa cime, annonciateur de Dionysos. L'écrivain est toujours en peine derrière son propre message qu'il ne sait comment dire, il n'est jamais à la hauteur de son devoir; Nerval le reconnaît ironiquement dans Promenades et souvenirs: "Livré souvent aux soins des domestiques et des paysans, j'avais nourri mon esprit de croyances bizarres, de légendes et de vieilles chansons. Il y avait là de quoi faire un poète, et je ne suis qu'un rêveur en prose » (III, 680-1) La quête d'un livre introuvable, suite au capharnaüm des bibliothèques, pour faire un livre infaisable, suite à une censure de l'époque, donne naissance à la nouvelle «Angélique » qui raconte précisément cette quête: tout en affirmant sa double impossibilité, elle s'engendre littérairement. Nerval définit sa nouvelle comme un «feuilleton-roman » interdit et frémit « en songeant à cette interprétation vague, qu'il serait possible de donner à ces deux mots bizarrement accouplés » (III, 461). Voilà la logique des créations de Nerval : un accouplement bizarre et paradoxal qui se crée négativement et par ce geste, veut régénérer du sens.

55 Dumas, évoquant la «folie» présente dans «El Desdichado», semble avoir bien compris la logique interne de l'imagination nervalienne, qui se confronte mélancoliquement à l'impuissance du langage : "l'imagination, cette folle du logis, en chasse [de l'esprit] momentanément la raison qui n'est que la maîtresse; alors, la première reste seule, toute puissante, dans ce cerveau nourri de rêves et d'hallucinations, [...] le jette dans les théories impossibles, dans les livres infaisables » (III, 449-50). Mais ce dont Nerval rend compte, c'est de la tâche même de la poésie de se confronter à un sens qui ne peut être dit que par une combinaison paradoxale d'éléments hétérogènes dont le lien seul fait émerger de nouvelles configurations sémantiques. Car tout en attestant de son échec, Nerval sublime son propre geste et ouvre un nouvel horizon poétique où se renouvelle le principe même de création, comme il le définit dans le Voyage en Orient :

56 Tu copies la nature avec froideur [...] Enfant, l'art n'est point là : il consiste à créer. [...] à coté de l'homme et des animaux existants, que ne cherches-tu de même des formes inconnues, des êtres innomés, des incarnations devant lesquelles l'homme a reculé, des accouplements terribles, des figures propres à répandre le respect, la gaieté, la stupeur et l'effroi ! (II, 652)

57 La mélancolie nous permet finalement de mieux saisir les tenants réels de la création chez Nerval, et par là peut-être de relativiser certains préjugés sur le romantisme, 
notamment français. Entre l'idée du génie total, ne prônant aucune autre valeur davantage que l'originalité, et l'image du nostalgique poète qui compose ses plaintes sur les ruines d'un temps perdu, la mélancolie, si l'on accepte de lui donner sa pleine mesure conceptuelle, inscrit l'art romantique dans un horizon quelque peu différent. Dans le temps et l'espace de la mélancolie, il n'y a plus événement, ni surgissement; le «nouveau » perd sa crédibilité et son attrait. Le passé est le champ qu'investigue le poète, non pour répéter ou ressasser une absence, mais précisément pour inventer et créer de nouvelles configurations poétiques. Comme le dit Nerval dans l'une de ses premières œuvres: "Les siècles écoulés se conservent tout entiers à l'état d'intelligences et d'ombres, dans une suite de régions concentriques, étendues à l'entour du monde matériel » (I, 503). Pour l'auteur, comme le note avec beaucoup de pertinence Hisashi Mizuno,

[...] la matière est en état de virtualité comme attendant une nouvelle reconstitution ; le néant peut être la source primitive de la vie. D'où vient un sentiment ambivalent chez Nerval à l'égard du monde décomposé ; d'une part, c'est le vide et la mort, d'autre part, tout vient de là et tout y retourne. Dans cette perspective, le monde réel n'est qu'une composition passagère faite avec des matières-débris qui ont repris vie dans le monde de l'âme après la désagrégation du monde précédent. (64)

59 La récurrence des ruines dans le romantisme n'est pas le signe d'une désolation du sens, mais celle d'une reprise : la défiguration du temps change l'objet mais ne l'annule pas, il le transforme en un espace ouvert à d'autres réseaux de signification. La mélancolie n'est pas un affaissement de la vie sur elle-même, mais au contraire une tension de celle-ci, comme le note Romano Guardini : « Le ressort qui est au cœur de la mélancolie, c'est Eros, le désir de l'amour et de la beauté » (57). Inventer d'autres beautés, de nouveaux régimes esthétiques avec les ruines d'anciens, et précisément au moyen de ces derniers, voilà peut-être comment le romantisme - Nerval à tout le moins - entend enregistrer la modernité de son geste poétique.

61 Aussi les poèmes des Chimères sont-ils écrits « dans cet état de rêverie supernaturaliste comme diraient les allemands ", ils « ne sont guère plus obscurs que la métaphysique d'Hegel [...] et perdraient leur charme à être expliqués, si la chose était possible » (III, 458). Cette dernière assertion peut être comprise ironiquement comme une invitation de Nerval à expliquer ses compositions et à tenter d'y trouver un sens qui n'y est pas moins présent que dans la philosophie allemande. Mais plus encore s'agit-il de les comprendre en d'autres termes que ceux d'une explicitation théorique: leur signification n'est pas isolable de leur forme. Le sens divin découvert au sein du rêve n'est pas représentable comme tel, il appartient intrinsèquement au régime de l'art, " c'est-à-dire un sens qui est mis en forme une fois, dans une physionomie singulière et non-répétable. » (Van Eynde 26) Cette singularité de la forme est nécessaire pour dire la mélancolie - non dire mélancoliquement ou parler de mélancolie -, et par là proposer un régime poétique du langage véritablement habité, traversé, hanté par le concept.

Pour conclure, il semble qu'une recherche axée sur le lien de l'œuvre et du concept a en effet le mérite d'un enrichissement réciproque non négligeable, et ce dernier s'organise autour de la question de la forme. D'une part, l'œuvre travaille le concept, l'idée, d'une autre façon que la philosophie, en réalité depuis une autre forme, qui l'incarne. Il se produit par là un décalage fécond entre définition, théorie, et une production artistique qui parvient sans doute à une performativité différente du sens 
que peut recouvrir le concept pour l'homme, tant dans son rapport à la création qu'à sa propre existence. D'autre part, le concept éclaire certaines des structures fondamentales de l'œuvre, ses enjeux philosophiques et la logique de sa création, en mettant en évidence, dans le cas ci-dessus présenté, la cohérence et la nécessité d'une forme, complètement inséparable du concept qu'elle sous-tend. Ce n'est bien sûr qu'à cette condition que l'œuvre des Chimères peut espérer performer, auprès de ses lecteurs ou auditeurs, le sens d'une mélancolie qui règle un certain régime de la création artistique et de l'être au monde de l'homme moderne, régime qui trouve son symbole dans la figure nervalienne du « soleil noir ».

\section{BIBLIOGRAPHIE}

Ouvrages cités

Brix, Michel. « Enjeux et significations de l'ésotérisme nervalien ». Verbum. Analecta neolatina. $\mathrm{N}^{\circ}$ $2: 173-182$.

Buci-Glucksmann, Christine. «Le cogito mélancolique de la modernité ». Magazine littéraire. $\mathrm{N}^{\circ}$ 244, $1987: 38-41$.

Chateaubriand, François-René (de). Cuuvres complètes. Ed. B. Didier. Paris : Champion, 2008.

Gilon, Odile. « Melancholia imaginativa ». Phantasia, N¹, 2015. Consulté le 19 septembre 2016, <http://popups.ulg.ac.be/0774-7136/index.php?id=356>.

Guardini, Romano. De la mélancolie. Trad. J. Ancelet-Hustache. Paris : Seuil, 1992.

Kristeva, Julia. Soleil noir. Dépression et mélancolie. Paris : Gallimard, 1987.

Laforgue, Pierre. «Soleil noir d'Aurélia », Revue d'Histoire littéraire de la France. 105, N4, 2005 : 843-857.

Marchal, Bertrand. « Notices, notes et variantes » in Nerval, Les Filles du feu. Les Chimères. Paris : Gallimard, $2005: 333-437$.

Mizuno, Hisashi. « Corps et âme dans Aurélia de Gérard de Nerval ». Romantisme. N¹27, 2005 : 59-77.

Nerval, Gérard (de). Euvres complètes. Ed. J. Guillaume et C. Pichois. Paris : Gallimard, bibliothèque de La Pléiade, 3 vol., 1984-93.

Raybaud, Antoine. « L'expansion mélancolique ». Magazine littéraire. № 244, 1987 : 46-7.

Staël, Germaine (de). De la Littérature. Ed. G. Gengembre et J. Goldzink, Paris : GarnierFlammarion, 1991.

Van Eynde, Laurent. « Eléments d'une épistémologie de la littérature ». Littérature et savoir(s), dir. S. Klimis, L. Van Eynde. Bruxelles : Publications des Facultés universitaires Saint-Louis, 2002 : 21-40. 


\section{NOTES}

1. Pierre Laforgue, "Soleil noir d'Aurélia », Revue d'Histoire littéraire de la France, 105, n4, 2005, p. 843-857.

2. "Je criai longtemps, invoquant ma mère, sous tous les noms donnés aux divinités antiques" (III, 754).

3. «La solitude absolue, le spectacle de la nature, me plongèrent bientôt dans un état presque impossible à décrire. Sans parents, sans amis, pour ainsi dire seul sur la terre, n'ayant point encore aimé, j'étais accablé par une surabondance de vie » (Chateaubriand 405).

4. Il s'agit, pour Henri de Gand, du risque de la mélancolie : elle permet certes une appréhension plus aisée des formes mais peut se laisser déborder par elles dans l'imagination. Le mélancolique délaisserait en conséquence la spéculation pour la poésie, mais aussi la fureur, les idées fixes... Cependant, seuls les anges seraient capables de penser totalement sans image, d'atteindre une pure réflexion métaphysique, ce qui poursuit le paradoxe de la mélancolie lorsqu'on songe que celle-ci, dans l'œuvre célèbre de Dürer, est justement représentée par un ange. Sur cette question, se référer à l'excellent article d'Odile Gilon sur le concept de mélancolie dans la tradition médiévale : Odile Gilon, « Melancholia imaginativa », Phantasia, vol. 1, 2015.

5. Concernant cette dimension optique de la mélancolie, voir Christine Buci-Glucksmann, « L'œil de la pensée. Une mélancolie tragique », Ecrit du temps, n¹3, 1987, p. 23-44.

6. Dans l'un de ses rêves, le narrateur d'Aurélia perçoit «ce bourdonnement monotone qui semble une prière à la déesse Mnémosyne » (III, 698).

\section{RÉSUMÉS}

Résumé

Dans cet article, je tente de montrer comment concept et œuvre poétique sont à même de s'éclairer l'un l'autre, et ce à partir de l'idée de mélancolie dans Les Chimères de Gérard de Nerval. La mélancolie apparaît comme un thème récurrent du recueil, lui donnant une cohérence et dévoilant la profondeur d'un questionnement existentiel qui hante toute l'écriture nervalienne. Réciproquement, le régime poétique du langage donne forme au concept, l'incarne dans les images et le rythme qui lui sont propres, et par là en développe la portée comme la signification. L'étude de la mélancolie chez Nerval révèle ultimement un mode de création proprement romantique, en quête d'un sens qui ne se donne qu'à travers un travail de l'imagination mobilisant les formes fantomatiques d'un passé en permanente recomposition.

\section{Abstract}

In this article, I will try to show how concepts and poetical works can shed light on each other, by studying the idea of melancholy in Gérard de Nerval's Les Chimères. Melancholy appears as a recurring theme in the volume of poetry, making the book coherent and revealing the depth of an existential question haunting the Nervalian writing. Conversely, the poetical regime of language gives substance to the concept. Indeed, it embodies representations and rhythm of its own, thus contributing to developing its meaning. The study of melancholy in Nerval's work ultimately reveals a mode of creation that is characteristically romantic, in search of a meaning which is only unveiled through a work of imagination mobilizing the ghostly forms of a past in perpetual rearrangement. 
INDEX

Mots-clés : Mots-clés

Keywords : Keywords 